\title{
Anxiety and depression in athletes assessed using the 12-item General Health Questionnaire (GHQ-12) - a systematic scoping review
}

\author{
N Armino,1,2 (iD BSc Honours; V Gouttebarge,1,3,4 iD PhD; S Mellalieu, $\mathbf{5}$ (iD PhD; R Schlebusch,6 iD PGCE Dip; JP van Wyk,6 (iD \\ ND Sports Management; S Hendricks $1,2,7$ iD $\mathrm{PhD}$ \\ ${ }^{1}$ Division of Exercise Science and Sports Medicine, Department of Human Biology, Faculty of Health Sciences, University of Cape Town, Cape Town, South \\ Africa \\ ${ }^{2}$ Health, Physical Activity, Lifestyle and Sport (HPALS) Research Centre, University of Cape Town, Cape Town, South Africa \\ ${ }^{3}$ Amsterdam UMC, University of Amsterdam, Department of Orthopaedic Surgery, Amsterdam Movement Sciences, Meibergdreef 9, Amsterdam, \\ Netherlands \\ ${ }^{4}$ Amsterdam Collaboration on Health \& Safety in Sports (ACHSS), Amsterdam UMC IOC Research Center of Excellence, Amsterdam, Netherlands \\ ${ }^{5}$ Cardiff School of Sport and Heath Sciences, Cardiff Metropolitan University, Cardiff, United Kingdom \\ ${ }^{6}$ South African Cricketers' Association, Cape Town, South Africa \\ ${ }^{7}$ Institute for Sport, Physical Activity and Leisure, Leeds Beckett University, Leeds, United Kingdom
}

Corresponding author: $S$ Hendricks (sharief.hendricks01@gmail.com)

Background: The poor mental health of athletes is a major concern in sport. Typically, the incidence/prevalence of mental health symptoms in athletes is studied using symptom-specific questionnaires. For symptoms of depression/anxiety, one such selfreporting questionnaire is the 12-item General Health Questionnaire (GHQ-12).

Objectives: The aim of this review was to synthesise and compare studies using the GHQ-12 in athletes to inform future research bodies by identifying trends and gaps in the literature.

Methods: A systematic search of five electronic databases (Google Scholar, PubMed, PsychINFO, Scopus and Web of Science) was conducted on all published studies up to 1 January 2019. Inclusion criteria: (1) participants were able-bodied athletes; (2) studies measured anxiety/depression using the GHQ-12; (3) studies were full original articles from peer-reviewed journals; (4) studies were published in English.

Results: Thirty-two studies were included in the review. The prevalence and incidence of symptoms of anxiety/depression ranged from $21-48 \%$ and $17-57 \%$, respectively. The majority of studies screening anxiety/depression using the GHQ-12 were cross-sectional. Almost $70 \%$ of the studies used the traditional scoring method. The majority of study populations sampled allmale cohorts comprising football (soccer) players.

Conclusion: The traditional scoring of $0-0-1-1$ should be used with the cut-off set at $\geq 3$. Also, the mean GHQ- 12 score should be reported. Potential risk factors for symptoms of anxiety/depression (i.e. recent adverse life events, injury and illness, social support, pressure to perform and career transitioning) and a lack of prospective studies were identified. Future research should also broaden the spectrum of athlete populations used and aim to improve response rates.

Keywords: mental health, elite athletes, sport, well-being

S Afr J Sports Med 2021;33:1-13. DOI: 10.17159/2078-516X/2021/v33i1a10679

Poor mental health of current and retired athletes is a major concern in sport. ${ }^{[1-7]}$ Typically, the incidence/prevalence of mental (1) health symptoms in athletes is studied using
symptom-specific questionnaires. For symptoms of anxiety and depression (often comorbid), one such self-reporting questionnaire is the 12-item General Health Questionnaire (GHQ-12). The GHQ-12 is a popular self-reporting measure of mental health. ${ }^{[8]}$ The GHQ has been used in community, clinical and sport settings. ${ }^{[9,10]}$ The original GHQ consists of 60 items, while the shorter more popular version contains only 12 items (GHQ-12). ${ }^{[11-13]}$ The popularity of the GHQ-12 can be attributed to its robust psychometric properties and being quick and unobtrusive to administer.[11-13] The GHQ-12 includes six positively phrased items (e.g. 'Have you been able to concentrate on what you were doing') and six negatively worded items (e.g. 'Have you lost much sleep over worry'). The GHQ-12 can be scored in several ways. For the traditional GHQ scoring method (0-0-1-1), items are answered on a 4-point scale with the response categories and scoring values for the positive items representing: 'not at all' $=1$, 'no more than usual'=1, 'rather more than $u^{\prime} u_{a l}=0$, and 'much more than usual' $=0$; and those for the negative items 'not at all' $=0$, 'no more than usual' $=0$, 'rather more than usual' $=1$, and 'much more than usual'=1.[14] The scores are summed to obtain a total score between $0-12$. A score of $\geq 2$ indicates symptoms of anxiety/depression. ${ }^{[9,11]}$ Goldberg also suggested that to obtain an optimal trade-off between sensitivity and specificity, the mean score of the group could be used as a threshold.[15] The less frequently used Likert-type scale scoring (0-1-2-3) can also be used. The scores are summed to obtain a total score between 0-36, with higher scores indicative of lower psychological wellbeing. Because of the larger scoring range compared to the traditional scoring method, the Likert-type scale scoring is potentially more sensitive in detecting changes in psychological well-being over time. ${ }^{[11]}$

While a number of reviews on the mental health of elite athletes are available in the literature, ${ }^{[2-5]}$ no review currently exists which focuses specifically on anxiety and depression using the GHQ-12. One narrative review provided an overview of the prevalence and risk factors for depression but failed to discuss the tools used to measure depression. ${ }^{[5]}$ Other reviews 
have a broad scope, covering many mental health symptoms and psychological well-being behaviours (e.g. sleep disorders, ADHD/ADD, eating disorders). ${ }^{[2-7]}$ This makes it difficult to compare studies to develop interventions for depression and anxiety. In response, a scoping review of the literature focusing on studies using the GHQ-12 was performed. The aim of this review was to synthesise and compare studies using the GHQ-12 in athletes in order to inform future research by identifying trends and gaps in the literature.

\section{Methods}

\section{Search}

This review was reported in accordance with the Preferred Reporting Items for Systematic Reviews and Meta-Analyses (PRISMA) guidelines. A systematic search of five electronic databases (Google Scholar, PubMed, PsychINFO, Scopus and Web of Science) was conducted on all published studies up to 1 January 2019. Key terms included: anxiety, depression, mental health, elite athletes, athletes, sport, general health questionnaires, GHQ and combinations thereof. Inclusion criteria for the review were: (1) participants were able-bodied athletes; (2) studies measured anxiety/depression using the GHQ-12; (3) studies were full, original articles from peerreviewed journals; (4) studies were published in English. Studies were excluded if athlete and non-athlete populations were combined as a single group. Athletes are defined in this review as individuals who train in sports aiming to improve their performance; are actively participating in sport competitions; registered in a local, regional or national sports federation; and devote several hours on most days to their sport. Studies were screened at the title and abstract level for eligibility. If a decision on the eligibility of the study was unclear at the title and abstract level, the full text was retrieved and screened. After merging the databases and removing duplicates, a second author screened the studies for reliability purposes. A schematic of the process is shown in Fig. 1.

\section{Data extraction}

The authors, year of publication, study design, purpose of the study, study population characteristics (sample size, age, active/retired, ratio of men to women, country), GHQ-12 (scoring method, cut-off point, mean score), prevalence and incidence of anxiety/depression, as well as factors associated with anxiety/depression were extracted from each study and tabulated.
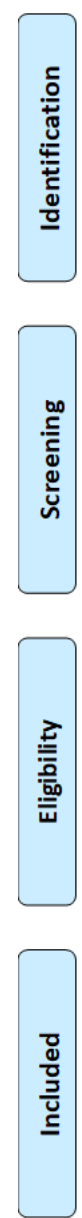

\section{Results}

A total of 202 studies were identified through database searching, while five were retrieved through other sources. After duplicates were removed, 175 studies were screened by title/abstract, of which 37 full texts were assessed. Finally, 32 studies were included in the review (Table 1).

\section{Study design}

The majority of studies used either a cross-sectional (56\%, $\mathrm{n}=18),{ }^{[9,16-32]}$ or a prospective design $(38 \%, \mathrm{n}=12) \cdot{ }^{[33-44]}$ One study used a randomised controlled trial design ${ }^{[45]}$ and another used a quasi-experimental design. ${ }^{[46]}$

\section{Population characteristics}

The majority of studies were conducted on football players $(50 \%, n=16)$. Other sports that have used the GHQ-12 are rugby union $(16 \%, n=5)$, cricket $(9 \%, n=3)$, ice hockey $(9 \%, n=3)$, and Gaelic football $(6 \%, n=2)$. Only one study has been published on Australian football players, distance runners, handball players, and horse jockeys, respectively. The majority of studies included all-male cohorts $(69 \%, n=22)$, while only one study included an all-female cohort. Several studies included mixed cohorts $(22 \%, n=7)$, while a couple did not specify the sex of the participants $(6 \%, n=2)$. The majority of studies included adult athletes $(81 \%, \mathrm{n}=26)$, while some used only adolescent athletes $(13 \%, n=4)$. A few studies included both adult and adolescent

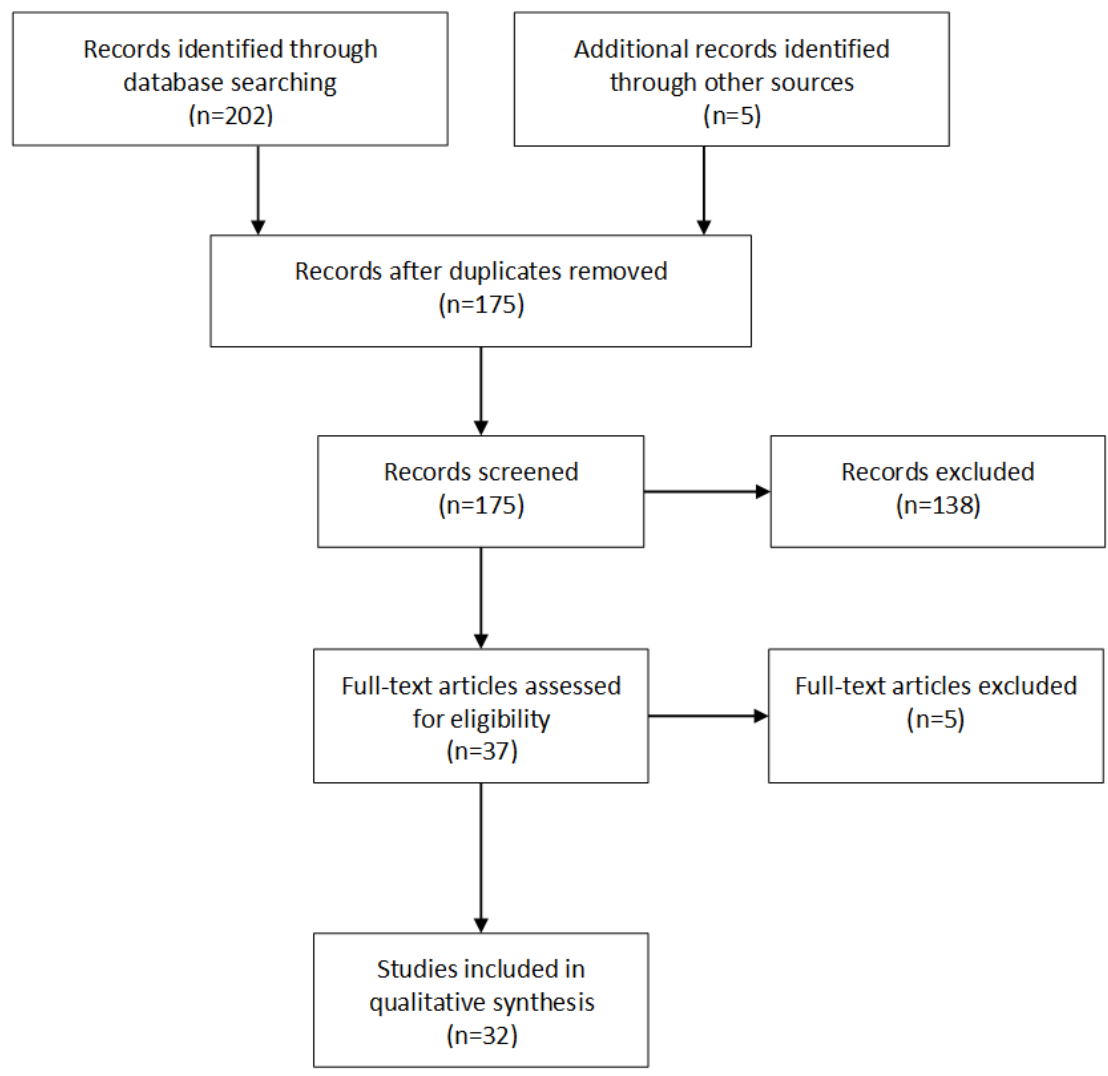

Fig. 1. Schematic of the literature search according to PRISMA guidelines 
athletes $(6 \%, n=2)$. Most of the studies consisted of active athletes $(63 \%, n=20)$. Several studies included retired athletes exclusively $(19 \%, n=6)$, while others included both active and retired athletes $(19 \%, n=6)$. Most of the included studies used elite athletes, where this was defined as professional, international/national or Olympic-level athletes $(84 \%, \mathrm{n}=27)$. Non-elite athletes in other studies included university, adolescent/high school, or local/regional athletes $(16 \%, n=5)$.

\section{Prevalence/incidence}

The prevalence of symptoms of anxiety/depression assessed by the GHQ-12 ranged from 21-48\%. The incidence of symptoms of anxiety/depression ranged from $17-57 \%$.

\section{GHQ scoring method and cut-off points}

The studies $(72 \%, \mathrm{n}=23)$ used mainly the 'traditional' GHQ scoring method (0-0-1-1), while $19 \%(n=6)$ used the Likertscale scoring method (0-1-2-3). The remaining studies $(9 \%$, $\mathrm{n}=3$ ) did not specify the GHQ scoring method. Of the 23 studies that used the traditional GHQ scoring method, $43 \%$ $(n=10)$ set the cut-off at $\geq$ two, another $43 \%(n=10)$ at $\geq 3$ and two studies ( $9 \%$ ) set the cut-off at $\geq 4$. One study did not specify the cut-off point for indicating symptoms of anxiety/depression.

\section{Discussion}

The GHQ-12 is a popular tool used to screen the presence of anxiety/depression symptoms among athletes. Its popularity can be attributed to its robust psychometric properties and quick unobtrusive administration. ${ }^{[11-13]}$ This is the first review to focus specifically on the GHQ-12 in order to compare studies and identify potential risk factors for anxiety/depression, as well as methodological considerations for future research. Not surprisingly, methodological inconsistencies between studies using the GHQ-12 were found. Sixty-eight percent $(n=23)$ of the studies used the traditional 0-0-1-1 scoring method. Of these 23 studies, three different cut-offs were applied. For future research, we recommend the traditional scoring of $0-0-1-1$ be used. Furthermore, to improve anxiety/depression prevalence and incidence comparisons, we also suggest that the recommended cut-off for the GHQ-12 for athletes be set at $\geq 3$. In addition, the mean GHQ-12 score should be reported, as suggested by Goldberg. ${ }^{[15]}$

Most of the studies used a cross-sectional or prospective cohort design with the objective of determining the prevalence and incidence of symptoms of anxiety/depression. The prevalence and incidence ranged from $21-48 \%$ and $17-$ $57 \%$, respectively. ${ }^{[9,16,17,19,23,25-34,36-40,42,44,46]}$ A sample of elite athletes from the United Kingdom (cricket, fencing, hockey, rugby union and many others) and elite Gaelic athletes presented with the highest prevalence of anxiety/depression symptoms $(48 \%),{ }^{[9,40]}$ while the highest incidence was found in a sample of elite Dutch athletes (57\%). ${ }^{[38]}$ The response rates for most of these epidemiological studies were around 30\%, with $40 \%$ comprising samples of European professional

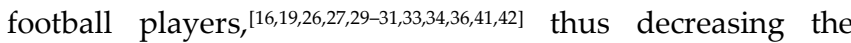
generalisability of the findings and highlighting a clear area for further investigation.

Several studies have identified potential risk factors for anxiety/depression symptoms. The most notable of these are recent adverse life events, ${ }^{[17,19,27,31,44]}$ career dissatisfaction, ${ }^{[9,26,44]}$

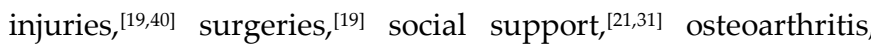
pressure to perform, and career transitioning. ${ }^{[1,23]}$ In one study, the GHQ score was used as a predictor for musculoskeletal injury. ${ }^{[42]}$ Although no reported association was found between anxiety/depression symptoms and severe musculoskeletal injuries, the study was novel in using the GHQ score as a potential risk factor for predicting injury. Using the GHQ score as a predictor rather than an outcome suggests that the GHQ12 could potentially be used as a monitoring tool for injury risk. Two studies attempted to reduce the GHQ-12 scores of athletes. Wilson et al. attempted to reduce GHQ-12 scores in 10 experienced jockeys using an exercise and diet intervention. ${ }^{[46]}$ However, the study was limited by its small sample size and lacked a control group. The other study used a randomised control study design, where the intervention was an internetbased cognitive behavioural therapy. ${ }^{[45]}$ No significant differences were found between the intervention and control group, and this was attributed to the short period of the intervention.

The GHQ-12 is proposed to measure symptoms of anxiety and depression (reported also as anxiety/depression). ${ }^{[2]}$ Although often comorbid, these are two different psychological conditions. Depression is a medical condition that negatively impacts on how an individual feels, thinks and acts. ${ }^{[4]}$ Symptoms include sadness, apathy, guilt, low self-esteem, trouble sleeping, decreased appetite, tiredness, poor concentration and suicidal ideation. ${ }^{[47]}$ Depression can be chronic or recurrent, and can significantly affect an individual's ability to cope with daily life.[48] Anxiety is defined as the anticipation of a future concern, whilst fear is an emotional response to an immediate threat. ${ }^{[47]}$ Anxiety disorders are characterised by excessive feelings of anxiety and fear. ${ }^{[48]}$ Researchers and sport practitioners should be aware of the distinctions between depression and anxiety when using the GHQ-12.

\section{Conclusion}

This review compared GHQ-12 studies and identified potential risk factors for depression and anxiety, as well as methodological considerations for future research. Based on this review, we recommend the traditional scoring of $0-0-1-1$ be used with the cut-off set at $\geq 3$. Also, the mean GHQ-12 score should be reported. The prevalence and incidence of symptoms of anxiety/depression ranged from $21-48 \%$ and $17-57 \%$, respectively. Potential risk factors for anxiety/depression include recent adverse life events, $[17,19,27,31,44]$ career dissatisfaction, ${ }^{9,26,44]}$ injuries, ${ }^{[19,40]}$ surgeries, ${ }^{[19]}$ social support, ${ }^{[21,31]}$ osteoarthritis, pressure to perform, and career transitioning.[21,23] Future research should broaden the spectrum of athlete populations and aim to improve response rates. Finally, researchers and sport practitioners should acknowledge that the GHQ-12 does not differentiate between symptoms of anxiety and depression. 
Table 1. Summary of General Health Questionnaire (GHQ) studies on athletes

\begin{tabular}{|c|c|c|c|c|c|c|c|c|c|c|c|c|}
\hline Author & Study design & Purpose & $\begin{array}{l}\text { Population; } \\
\text { Country }\end{array}$ & $\begin{array}{l}\text { N; Men/ } \\
\text { Women \% }\end{array}$ & $\begin{array}{l}\text { GHQ } \\
\text { scoring } \\
\text { method }\end{array}$ & $\begin{array}{l}\text { GHQ } \\
\text { cut off } \\
\text { point }\end{array}$ & Prevalence & Incidence & Predictor & Outcome & $\begin{array}{l}\text { GHQ } \\
\text { mean } \\
\text { score } \\
\end{array}$ & Findings \\
\hline $\begin{array}{l}\text { Blakelock et } \\
\text { al., 2016 }\end{array}$ & $\begin{array}{l}\text { Observational, } \\
\text { prospective } \\
\text { cohort }\end{array}$ & $\begin{array}{l}\text { Psychological } \\
\text { distress } \\
\text { following } \\
\text { deselection in } \\
\text { soccer players }\end{array}$ & $\begin{array}{l}\text { Elite adolescent } \\
\text { footballers, 15-18 } \\
\text { years }(16.31 \pm 1.10) \text {; } \\
\text { UK }\end{array}$ & $91 ; 100 / 0$ & $\begin{array}{l}\text { GHQ } \\
\text { method }\end{array}$ & 3 & $\begin{array}{l}36 \% \text { (time } \\
\text { point } 1 ; \\
\mathrm{n}=14 \text { ) }\end{array}$ & $\begin{array}{l}55 \% \text { (time } \\
\text { point 3; } \\
\mathrm{n}=11 \text { ) }\end{array}$ & - & - & $\begin{array}{l}\text { At times } \\
1,2,3 \text { for } \\
\text { released } \\
\text { players: } \\
2.64 ; \\
3.82 ; \\
3.36 \text {. }\end{array}$ & $\begin{array}{l}\text { Proportion of deselected } \\
\text { players experienced higher } \\
\text { levels of psychological distress } \\
\text { than retained players at } \\
\text { postselection time points; } \\
\text { psychological distress was } \\
\text { reduced in retained players } \\
\text { over time and did not change } \\
\text { in released players at } \\
\text { postselection time points. }\end{array}$ \\
\hline $\begin{array}{l}\text { Brown et al., } \\
2017^{[25]}\end{array}$ & $\begin{array}{l}\text { Observational, } \\
\text { cross-sectional }\end{array}$ & $\begin{array}{l}\text { Compare } \\
\text { mental health } \\
\text { between } \\
\text { former } \\
\text { professional } \\
\text { rugby players } \\
\text { who were and } \\
\text { weren't forced } \\
\text { to retire }\end{array}$ & $\begin{array}{l}\text { Retired profession } \\
\text { al rugby union } \\
\text { players; Age ( } 38 \pm \\
\text { 5); Age at } \\
\text { retirement }(31 \pm 4) \text {; } \\
\text { Career length }(8.9 \pm \\
\text { 3.8); Retirement }(7.4 \\
\pm 4.4) ; \text { France, Ire } \\
\text { land, South Africa }\end{array}$ & $\begin{array}{l}293: 173 \\
\text { (Voluntary } \\
\text { retirement), } \\
120 \text { (Forced } \\
\text { retirement); } \\
100 / 0\end{array}$ & NS & NS & $\begin{array}{l}29 \% \\
\text { (overall); } \\
26 \% \text { (retired } \\
\text { voluntarily); } \\
32 \% \text { (forced } \\
\text { retirement) }\end{array}$ & & - & - & - & $\begin{array}{l}\text { The overall prevalence for } \\
\text { anxiety/depression was } 29 \% \text {. } \\
\text { The prevalence for forced } \\
\text { retirement was } 32 \% \text {; and } 26 \% \\
\text { for voluntary retirement. The } \\
\text { prevalence between forced } \\
\text { retirement and voluntary } \\
\text { retirement was not } \\
\text { significantly different. }\end{array}$ \\
\hline $\begin{array}{l}\text { Foskett et al., } \\
2017^{[9]}\end{array}$ & $\begin{array}{l}\text { Observational, } \\
\text { cross-sectional }\end{array}$ & $\begin{array}{l}\text { Prevalence of } \\
\text { signs of } \\
\text { anxiety/depres } \\
\text { sion and } \\
\text { distress }\end{array}$ & $\begin{array}{l}\text { Sample of elite } \\
\text { athletes from } \\
\text { various individual } \\
\text { and team sports } \\
\text { competing at a } \\
\text { professional, } \\
\text { international or } \\
\text { national level; Age } \\
(24 \pm 8.6) \text {; UK }\end{array}$ & $\begin{array}{l}143 ; 57 / 43(1 \\
\text { participant } \\
\text { did not } \\
\text { specify } \\
\text { gender) }\end{array}$ & $\begin{array}{l}\text { GHQ } \\
\text { method }\end{array}$ & 2 & $48 \%$ & & $\begin{array}{l}\text { Career } \\
\text { dissatisfaction } \\
\text { *; severe } \\
\text { injuries }\end{array}$ & $\begin{array}{l}\text { GHQ } \\
\text { score }\end{array}$ & - & $\begin{array}{l}48 \% \text { prevalence of } \\
\text { anxiety/depression; career } \\
\text { dissatisfaction was a significant } \\
\text { independent predictor of signs } \\
\text { of anxiety/depression. }\end{array}$ \\
\hline $\begin{array}{l}\text { Gouttebarge } \\
\text { et al., 2015a } \mathbf{a}^{[31]}\end{array}$ & $\begin{array}{l}\text { Observational, } \\
\text { cross-sectional }\end{array}$ & $\begin{array}{l}\text { Mental and } \\
\text { psychosocial } \\
\text { health }\end{array}$ & $\begin{array}{l}\text { Current (mean age } \\
27 \pm 5 \text { ) and former } \\
\text { footballers (mean } \\
\text { age } 36 \pm 5) \text {; } \\
\text { duration of career: } \\
\text { current }(9 \pm 5) ; \\
\text { former }(12 \pm 5) ; \\
\text { duration of } \\
\text { retirement ( } 5 \pm 3 \text { ); } \\
60 \% \text { play/played in } \\
\text { the highest leagues; } \\
\text { Australia, Ireland, } \\
\text { The Netherlands, } \\
\text { New Zealand, } \\
\text { Scotland and USA }\end{array}$ & $\begin{array}{l}\text { 253: } 149 \\
\text { (current) } \\
\text { and } 104 \\
\text { (former); } \\
100 / 0\end{array}$ & $\begin{array}{l}\text { GHQ } \\
\text { method }\end{array}$ & 2 & $\begin{array}{l}26 \% \\
\text { (current); } \\
39 \% \text { (retired) }\end{array}$ & & $\begin{array}{l}\text { Severe } \\
\text { injuries; } \\
\text { surgeries; LE } \\
<12^{*} \text {; LE >12; } \\
\text { low social } \\
\text { support from } \\
\text { trainer/ } \\
\text { supervisor; } \\
\text { low social } \\
\text { support from } \\
\text { teammates/col } \\
\text { leagues* }\end{array}$ & $\begin{array}{l}\text { GHQ } \\
\text { score }\end{array}$ & - & $\begin{array}{l}26 \% \text { and } 39 \% \text { prevalence of } \\
\text { anxiety/depression in current } \\
\text { and former football players } \\
\text { respectively. Recent adverse } \\
\text { life events were significantly } \\
\text { associated with } \\
\text { anxiety/depression in current } \\
\text { and former footballers; low } \\
\text { social support from teammates } \\
\text { was also significantly } \\
\text { associated with } \\
\text { anxiety/depression in current } \\
\text { footballers. }\end{array}$ \\
\hline
\end{tabular}


Table 1 continued. Summary of General Health Questionnaire (GHQ) studies on athletes

\begin{tabular}{|c|c|c|c|c|c|c|c|c|c|c|c|c|}
\hline Author & Study design & Purpose & $\begin{array}{l}\text { Population; } \\
\text { Country }\end{array}$ & $\begin{array}{l}\text { N; Men/ } \\
\text { Women \% }\end{array}$ & $\begin{array}{l}\text { GHQ } \\
\text { scoring } \\
\text { method }\end{array}$ & $\begin{array}{l}\text { GHQ } \\
\text { cut off } \\
\text { point }\end{array}$ & Prevalence & Incidence & Predictor & Outcome & $\begin{array}{l}\text { GHQ } \\
\text { mean } \\
\text { score }\end{array}$ & Findings \\
\hline $\begin{array}{l}\text { Gouttebarge } \\
\text { et al., 2015b }\end{array}$ & $\begin{array}{l}\text { Observational, } \\
\text { cross-sectional }\end{array}$ & $\begin{array}{l}\text { Prevalence } \\
\text { and } \\
\text { determinants } \\
\text { of symptoms } \\
\text { related to } \\
\text { mental } \\
\text { disorders }\end{array}$ & $\begin{array}{l}\text { Retired } \\
\text { professional } \\
\text { footballers; mean } \\
\text { age } 35 \text {; duration of } \\
\text { career }(12 \pm 5) \text {; } \\
\text { duration of } \\
\text { retirement }(4 \pm 4) \text {; } \\
\text { various countries }\end{array}$ & $219 ; 100 / 0$ & $\begin{array}{l}\text { GHQ } \\
\text { method }\end{array}$ & 2 & $35 \%$ & & $\begin{array}{l}\text { Severe injuries; } \\
\text { surgeries; LE } \\
<6^{*} ; \text { LE }>6 ; \\
\text { career } \\
\text { dissatisfaction }\end{array}$ & $\begin{array}{l}\text { GHQ } \\
\text { score }\end{array}$ & - & $\begin{array}{l}35 \% \text { prevalence of } \\
\text { anxiety/depression. } \\
\text { Significant positive } \\
\text { association of recent life } \\
\text { events with } \\
\text { anxiety/depression. }\end{array}$ \\
\hline $\begin{array}{l}\text { Gouttebarge } \\
\text { et al., 2015c } c^{[30]}\end{array}$ & $\begin{array}{l}\text { Observational, } \\
\text { cross-sectional }\end{array}$ & $\begin{array}{l}\text { Symptoms of } \\
\text { common } \\
\text { mental } \\
\text { disorders in } \\
\text { professional } \\
\text { football }\end{array}$ & $\begin{array}{l}\text { Professional } \\
\text { footballers; } \\
\text { Finland, France, } \\
\text { Norway, Spain, } \\
\text { Sweden }\end{array}$ & $\begin{array}{l}540: 121 \\
\text { (Finland), } 81 \\
\text { (France), } 119 \\
\text { (Norway), } \\
70 \text { (Spain), } \\
149 \\
\text { (Sweden); } \\
100 / 0\end{array}$ & $\begin{array}{l}\text { GHQ } \\
\text { method }\end{array}$ & 2 & $25-43 \%$ & - & $\begin{array}{l}\text { LE }<6 \text {; LE }>6 ; \\
\text { career } \\
\text { dissatisfaction }\end{array}$ & $\begin{array}{l}\text { GHQ } \\
\text { score }\end{array}$ & - & $\begin{array}{l}25-43 \% \text { prevalence of } \\
\text { anxiety/depression (Spain- } \\
\text { Norway); Significant } \\
\text { correlation of life events and } \\
\text { career dissatisfaction with } \\
\text { anxiety/depression (Finland, } \\
\text { Sweden). }\end{array}$ \\
\hline $\begin{array}{l}\text { Gouttebarge } \\
\text { et al., 2015d } \mathrm{d}^{[16]}\end{array}$ & $\begin{array}{l}\text { Observational, } \\
\text { cross-sectional }\end{array}$ & $\begin{array}{l}\text { Severe } \\
\text { musculoskelet } \\
\text { al injuries and } \\
\text { symptoms of } \\
\text { CMD }\end{array}$ & $\begin{array}{l}\text { Professional } \\
\text { footballers (mean } \\
\text { age } 27 ; 54 \% \\
\text { playing in the } \\
\text { highest } \\
\text { professional } \\
\text { leagues); Finland, } \\
\text { France, Norway, } \\
\text { Spain, Sweden }\end{array}$ & $540 ; 100 / 0$ & $\begin{array}{l}\text { GHQ } \\
\text { method }\end{array}$ & 2 & $37 \%$ & - & $\begin{array}{l}\text { Severe injuries; } \\
\text { severe joint } \\
\text { injuries; severe } \\
\text { muscle injuries; } \\
\text { surgeries }\end{array}$ & $\begin{array}{l}\text { GHQ } \\
\text { score }\end{array}$ & - & $\begin{array}{l}37 \% \text { prevalence of } \\
\text { anxiety/depression. The } \\
\text { number of severe } \\
\text { musculoskeletal injuries } \\
\text { positively correlated with } \\
\text { anxiety/depression. } \\
\text { However, no significant } \\
\text { associations with severe } \\
\text { injuries/surgeries and } \\
\text { anxiety/depression. }\end{array}$ \\
\hline $\begin{array}{l}\text { Gouttebarge } \\
\text { et al., 2015e } \mathbf{e}^{[26]}\end{array}$ & $\begin{array}{l}\text { Observational, } \\
\text { prospective } \\
\text { cohort }\end{array}$ & $\begin{array}{l}\text { Symptoms of } \\
\text { common } \\
\text { mental } \\
\text { disorders and } \\
\text { adverse health } \\
\text { behaviours }\end{array}$ & $\begin{array}{l}\text { Professional } \\
\text { footballers (mean } \\
\text { age } 27 ; 55 \% \\
\text { playing in the } \\
\text { highest } \\
\text { professional } \\
\text { leagues); various } \\
\end{array}$ & $607 ; 100 / 0$ & $\begin{array}{l}\text { GHQ } \\
\text { method }\end{array}$ & 2 & $38 \%$ & - & $\begin{array}{l}\text { Severe injuries; } \\
\text { surgeries; LE } \\
<6 \text {; LE }>6 \text {; } \\
\text { career } \\
\text { dissatisfaction* }\end{array}$ & $\begin{array}{l}\text { GHQ } \\
\text { score }\end{array}$ & - & $\begin{array}{l}\text { Prevalence of } 38 \% \text { for } \\
\text { anxiety/depression. } \\
\text { Statistically significant } \\
\text { correlations were found for } \\
\text { severe injuries and career } \\
\text { dissatisfaction with } \\
\text { anxiety/depression. }\end{array}$ \\
\hline $\begin{array}{l}\text { Gouttebarge } \\
\text { et al., 2016a } \mathbf{a}^{[17]}\end{array}$ & $\begin{array}{l}\text { Observational, } \\
\text { cross-sectional }\end{array}$ & $\begin{array}{l}\text { Prevalence } \\
\text { and } \\
\text { determinants } \\
\text { of symptoms } \\
\text { of CMD }\end{array}$ & $\begin{array}{l}\text { Retired } \\
\text { professional } \\
\text { rugby union } \\
\text { players; mean: age } \\
\text { 38; career length 9; } \\
\text { retirement } \\
\text { duration 8; } \\
\text { France, Ireland } \\
\text { and South Africa }\end{array}$ & $295 ; 100 / 0$ & $\begin{array}{l}\text { GHQ } \\
\text { method }\end{array}$ & 2 & $28 \%$ & - & $\begin{array}{l}\mathrm{LE}<6^{*} ; \mathrm{LE}>6 ; \\
\text { career } \\
\text { dissatisfaction }\end{array}$ & $\begin{array}{l}\text { GHQ } \\
\text { score }\end{array}$ & - & $\begin{array}{l}\text { Anxiety/depression } \\
\text { prevalence of } 28 \% \text {; a higher } \\
\text { number of adverse life } \\
\text { events was associated with } \\
\text { anxiety/ depression. } \\
\text { Significant negative } \\
\text { correlation with career } \\
\text { dissatisfaction and anxiety/ } \\
\text { depression. }\end{array}$ \\
\hline
\end{tabular}




\section{Table 1 continued. Summary of General Health Questionnaire (GHQ) studies on athlete}

\begin{tabular}{|c|c|c|c|c|c|c|c|c|c|c|c|c|}
\hline Author & Study design & Purpose & $\begin{array}{l}\text { Population; } \\
\text { Country }\end{array}$ & $\begin{array}{l}\text { N; Men/ } \\
\text { Women \% }\end{array}$ & $\begin{array}{l}\text { GHQ } \\
\text { scoring } \\
\text { method }\end{array}$ & $\begin{array}{l}\text { GHQ } \\
\text { cut off } \\
\text { point } \\
\end{array}$ & Prevalence & Incidence & Predictor & Outcome & $\begin{array}{l}\text { GHQ } \\
\text { mean } \\
\text { score }\end{array}$ & Findings \\
\hline $\begin{array}{l}\text { Gouttebarge } \\
\text { et al., 2016b } \\
\text { [34] }\end{array}$ & $\begin{array}{l}\text { Observational, } \\
\text { prospective } \\
\text { cohort }\end{array}$ & $\begin{array}{l}\text { Symptoms } \\
\text { of CMD }\end{array}$ & $\begin{array}{l}\text { Professional } \\
\text { footballers (mean } \\
\text { age } 27 \text {; mean career } \\
\text { duration of } 8 \text { years; } \\
55 \% \text { playing in the } \\
\text { highest leagues) }\end{array}$ & $\begin{array}{l}84 \text { at } \\
\text { baseline and } \\
262 \text { at } \\
\text { follow up; } \\
100 / 0\end{array}$ & $\begin{array}{l}\text { GHQ } \\
\text { method }\end{array}$ & 3 & $32 \%$ & $37 \%$ & $\begin{array}{l}\text { Adverse life } \\
\text { events; } \\
\text { conflict with } \\
\text { trainer/coach; } \\
\text { career } \\
\text { dissatisfaction }\end{array}$ & $\begin{array}{l}\text { GHQ } \\
\text { score }\end{array}$ & - & $\begin{array}{l}\text { At baseline, there was a } 32 \% \\
\text { prevalence of anxiety/ } \\
\text { depression; there was a 12- } \\
\text { month incidence of } 37 \% \\
\text { anxiety/depression. Although } \\
\text { not statistically significant, } \\
\text { there was an association } \\
\text { between adverse life events } \\
\text { and career dissatisfaction with } \\
\text { anxiety/depression. }\end{array}$ \\
\hline $\begin{array}{l}\text { Gouttebarge } \\
\text { et al., 2016c }\end{array}$ & $\begin{array}{l}\text { Observational, } \\
\text { cross-sectional }\end{array}$ & $\begin{array}{l}\text { Relationship } \\
\text { of level of } \\
\text { education } \\
\text { and } \\
\text { employment } \\
\text { with } \\
\text { symptoms } \\
\text { of common } \\
\text { mental } \\
\text { disorders }\end{array}$ & $\begin{array}{l}\text { Current (mean age } \\
\text { 27) and Retired } \\
\text { (mean age 35) } \\
\text { professional } \\
\text { footballers; career } \\
\text { duration: current } \\
(7.8 \pm 4.4) \text {; retired } \\
(11.6 \pm 5.0) \text {; Belgium, } \\
\text { Chile, Finland, } \\
\text { France, Japan, } \\
\text { Norway, Paraguay, } \\
\text { Peru, Spain, Sweden } \\
\text { and Switzerland }\end{array}$ & $\begin{array}{l}607 \text { current } \\
\text { and } 219 \\
\text { retired; } \\
100 / 0\end{array}$ & $\begin{array}{l}\mathrm{GHQ} \\
\text { method }\end{array}$ & 2 & $\begin{array}{l}38 \% \\
\text { (current); } \\
35 \% \\
\text { (retired) }\end{array}$ & - & $\begin{array}{l}\text { Level of } \\
\text { education; } \\
\text { employment } \\
\text { status; } \\
\text { working } \\
\text { hours }\end{array}$ & $\begin{array}{l}\text { GHQ } \\
\text { score }\end{array}$ & - & $\begin{array}{l}\text { Anxiety/depression prevalence } \\
\text { of } 38 \% \text { and } 35 \% \text { among current } \\
\text { and retired football players } \\
\text { respectively. Significant } \\
\text { negative correlations between } \\
\text { employment status and } \\
\text { number of hours work with } \\
\text { anxiety /depression among } \\
\text { retired players. }\end{array}$ \\
\hline $\begin{array}{l}\text { Gouttebarge } \\
\text { et al., 2016d } \\
{\left[\begin{array}{l}{[32]}\end{array}\right.}\end{array}$ & $\begin{array}{l}\text { Observational, } \\
\text { cross-sectional }\end{array}$ & $\begin{array}{l}\text { Prevalence } \\
\text { and risk } \\
\text { indicators of } \\
\text { symptoms } \\
\text { of common } \\
\text { mental } \\
\text { disorders }\end{array}$ & $\begin{array}{l}\text { Current (mean: age } \\
\text { 27; career duration } \\
\text { 8) and former } \\
\text { (mean: age 50; career } \\
\text { duration 11; } \\
\text { retirement duration } \\
\text { 20) elite athletes } \\
\text { from various sports; } \\
\text { The Netherlands }\end{array}$ & $\begin{array}{l}485: 203 \\
\text { (current) } \\
\text { and } 282 \\
\text { (former); } \\
36 / 64 \\
\text { (current); } \\
49 / 51 \\
\text { (former) }\end{array}$ & $\begin{array}{l}\text { GHQ } \\
\text { method }\end{array}$ & 3 & $\begin{array}{l}45 \% \\
\text { (current); } \\
29 \% \\
\text { (former) }\end{array}$ & - & $\begin{array}{l}\text { Severe } \\
\text { injuries; } \\
\text { surgeries; } \\
\text { recent adverse } \\
\text { life events; } \\
\text { career } \\
\text { dissatisfaction } \\
\text {; support }\end{array}$ & $\begin{array}{l}\text { GHQ } \\
\text { score }\end{array}$ & - & $\begin{array}{l}45 \% \text { and } 29 \% \text { prevalence of } \\
\text { anxiety/depression among } \\
\text { current and former athletes } \\
\text { respectively. Current and } \\
\text { former athletes with a higher } \\
\text { number of severe injuries, } \\
\text { higher number of surgeries, } \\
\text { higher number of recent } \\
\text { adverse life events, higher level } \\
\text { of career dissatisfaction will be } \\
\text { more likely to report symptoms } \\
\text { of anxiety/depression. }\end{array}$ \\
\hline $\begin{array}{l}\text { Gouttebarge } \\
\text { et al., 2016e } \mathbf{e}^{[40]}\end{array}$ & $\begin{array}{l}\text { Observational, } \\
\text { prospective } \\
\text { cohort }\end{array}$ & $\begin{array}{l}\text { Epidemiolo } \\
\text { gy of CMD }\end{array}$ & $\begin{array}{l}\text { Elite Gaelic athletes } \\
\text { (hurlers and } \\
\text { footballers); mean: } \\
\text { age 25; career } \\
\text { duration 5; Ireland }\end{array}$ & $\begin{array}{l}204 \text { at } \\
\text { baseline and } \\
108 \text { at } \\
\text { follow up; } \\
100 / 0\end{array}$ & $\begin{array}{l}\text { GHQ } \\
\text { method }\end{array}$ & 3 & $48 \%$ & $21 \%$ & $\begin{array}{l}\text { Severe injury*; } \\
\text { surgeries; } \\
\text { recent life } \\
\text { events; career } \\
\text { dissatisfaction }\end{array}$ & $\begin{array}{l}\text { GHQ } \\
\text { score }\end{array}$ & - & $\begin{array}{l}48 \% \text { prevalence for } \\
\text { anxiety/depression; 6-month } \\
\text { incidence of } 21 \% \text { for anxiety/ } \\
\text { depression. Significant } \\
\text { association with severe injury } \\
\text { and the 6-month incidence for } \\
\text { anxiety/depression. }\end{array}$ \\
\hline
\end{tabular}




\section{REVIEW}

Table 1 continued. Summary of General Health Questionnaire (GHQ) studies on athletes

\begin{tabular}{|c|c|c|c|c|c|c|c|c|c|c|c|c|}
\hline Author & Study design & Purpose & $\begin{array}{l}\text { Population; } \\
\text { Country }\end{array}$ & $\begin{array}{l}\mathrm{N} ; \mathrm{Men} / \\
\text { Women \% }\end{array}$ & $\begin{array}{l}\text { GHQ } \\
\text { scoring } \\
\text { method }\end{array}$ & $\begin{array}{l}\text { GHQ } \\
\text { cut off } \\
\text { point }\end{array}$ & Prevalence & Incidence & Predictor & Outcome & $\begin{array}{l}\text { GHQ } \\
\text { mean } \\
\text { score }\end{array}$ & Findings \\
\hline $\begin{array}{l}\text { Gouttebarge } \\
\text { et al., 2017a } \mathbf{a}^{[38]}\end{array}$ & $\begin{array}{l}\text { Observational, } \\
\text { prospective } \\
\text { cohort }\end{array}$ & $\begin{array}{l}\text { Symptoms } \\
\text { of common } \\
\text { mental } \\
\text { disorders }\end{array}$ & $\begin{array}{l}\text { Elite athletes from } \\
\text { various sports; mean } \\
\text { age } 27 \text { years; mean } \\
\text { career duration } 8 \\
\text { years; The } \\
\text { Netherlands }\end{array}$ & $\begin{array}{l}203 \text { at } \\
\text { baseline } \\
\text { and } 143 \text { at } \\
\text { follow up; } \\
36 / 64\end{array}$ & $\begin{array}{l}\text { GHQ } \\
\text { method }\end{array}$ & 3 & $45 \%$ & $57 \%$ & $\begin{array}{l}\text { Being injured; } \\
\text { recent adverse } \\
\text { life events; } \\
\text { career } \\
\text { dissatisfaction }\end{array}$ & $\begin{array}{l}\text { GHQ } \\
\text { score }\end{array}$ & - & $\begin{array}{l}\text { At baseline, there was a } 45 \% \\
\text { prevalence of anxiety/depression; } \\
12 \text {-month incidence of } \\
\text { anxiety/depression was } 57 \% \text {. No } \\
\text { statistically significant } \\
\text { associations, but career } \\
\text { dissatisfaction did increase the } \\
\text { likelihood of symptoms of } \\
\text { anxiety/depression by } 3.5 \text { times. }\end{array}$ \\
\hline $\begin{array}{l}\text { Gouttebarge } \\
\text { et al., 2017b }\end{array}$ & $\begin{array}{l}\text { Observational, } \\
\text { prospective } \\
\text { cohort }\end{array}$ & $\begin{array}{l}\text { Symptoms } \\
\text { of common } \\
\text { mental } \\
\text { disorders }\end{array}$ & $\begin{array}{l}\text { Current (mean age } \\
26 ; \text { career duration } 8 \\
\text { years) and retired } \\
\text { (mean age } 35 \text {; career } \\
\text { duration } 11 \text { years) } \\
\text { professional ice } \\
\text { hockey players }\end{array}$ & $\begin{array}{l}258 \text { (135 } \\
\text { current and } \\
123 \text { retired } \\
\text { players) at } \\
\text { baseline } \\
\text { and } 158 \text { ( } 81 \\
\text { current and } \\
77 \text { retired } \\
\text { players) at } \\
\text { follow up; } \\
100 / 0\end{array}$ & $\begin{array}{l}\text { GHQ } \\
\text { method }\end{array}$ & 3 & $\begin{array}{l}24 \% \\
\text { (current); } \\
19 \% \text { (retired) }\end{array}$ & $\begin{array}{l}17 \% \\
\text { (current); } \\
8 \% \\
\text { (retired) }\end{array}$ & $\begin{array}{l}\text { Severe injuries; } \\
\text { surgeries; } \\
\text { recent adverse } \\
\text { life events; } \\
\text { career } \\
\text { dissatisfaction; } \\
\text { support }\end{array}$ & $\begin{array}{l}\text { GHQ } \\
\text { score }\end{array}$ & - & $\begin{array}{l}\text { There was a prevalence of } 24 \% \\
\text { and } 19 \% \text { of anxiety/depression for } \\
\text { current and retired athletes } \\
\text { respectively. The incidence of } \\
\text { symptoms of anxiety/ depression } \\
\text { was } 17 \% \text { and } 8 \% \text { for current and } \\
\text { retired athletes respectively. } \\
\text { Although not statistically } \\
\text { significant, severe injuries, recent } \\
\text { adverse life events and career } \\
\text { dissatisfaction increased the } \\
\text { likelihood of reporting symptoms } \\
\text { of anxiety/depression in current } \\
\text { and retired ice hockey players. }\end{array}$ \\
\hline $\begin{array}{l}\text { Gouttebarge } \\
\text { et al., 2017c } \mathrm{c}^{[28]}\end{array}$ & $\begin{array}{l}\text { Observational, } \\
\text { cross-sectional }\end{array}$ & $\begin{array}{l}\text { A history of } \\
\text { concussions } \\
\text { is associated } \\
\text { with } \\
\text { symptoms } \\
\text { of common } \\
\text { mental } \\
\text { disorders }\end{array}$ & $\begin{array}{l}\text { Former professional } \\
\text { athletes (football, ice } \\
\text { hockey and rugby } \\
\text { union); mean: age } \\
\text { 37; career duration } \\
\text { 10; years retired 7; } \\
\text { Finland, France, } \\
\text { Ireland, Norway, } \\
\text { South Africa, Spain, } \\
\text { Sweden, } \\
\text { Switzerland }\end{array}$ & $576 ; 100 / 0$ & $\begin{array}{l}\text { GHQ } \\
\text { method }\end{array}$ & 3 & $\begin{array}{l}26 \% \text { (whole } \\
\text { group); } 26 \% \\
\text { (football); } \\
18 \% \text { (ice } \\
\text { hockey); } \\
28 \% \text { (rugby) }\end{array}$ & - & $\begin{array}{l}\text { Number of } \\
\text { concussions }\end{array}$ & $\begin{array}{l}\text { GHQ } \\
\text { score }\end{array}$ & - & $\begin{array}{l}\text { Whole group prevalence of } 26 \% \\
\text { anxiety/depression; } 26 \%, 18 \% \text { and } \\
28 \% \text { prevalence anxiety/ } \\
\text { depression for football, ice hockey } \\
\text { and rugby players respectively. } \\
\text { There was a significant difference } \\
\text { in the number of concussions } \\
\text { when groups were divided by } \\
\text { presence/absence of symptoms of } \\
\text { anxiety/depression (presence } \\
\text { having a higher number of } \\
\text { concussions). Former athletes } \\
\text { reporting } 4 \text { or } 5 \text { concussions were } \\
\text { nearly } 1.5 \text { times as likely to report } \\
\text { symptoms of anxiety/depression; } \\
\text { those reporting } 6 \text { or more were } \\
\text { two times as likely to report } \\
\text { symptoms. This relationship was } \\
\text { found across all three sports } \\
\text { individually as well. }\end{array}$ \\
\hline
\end{tabular}


Table 1 continued. Summary of General Health Questionnaire (GHQ) studies on athletes

\begin{tabular}{|c|c|c|c|c|c|c|c|c|c|c|c|c|}
\hline Author & $\begin{array}{l}\text { Study } \\
\text { design }\end{array}$ & Purpose & $\begin{array}{l}\text { Population; } \\
\text { Country }\end{array}$ & $\begin{array}{l}\text { N; Men/ } \\
\text { Women \% }\end{array}$ & $\begin{array}{l}\text { GHQ } \\
\text { scoring } \\
\text { method }\end{array}$ & $\begin{array}{l}\text { GHQ } \\
\text { cut off } \\
\text { point }\end{array}$ & Prevalence & Incidence & Predictor & Outcome & $\begin{array}{l}\text { GHQ mean } \\
\text { score }\end{array}$ & Findings \\
\hline $\begin{array}{l}\text { Gouttebarge } \\
\text { et al., 2018 }\end{array}$ & $\begin{array}{l}\text { Observation } \\
\text { al, } \\
\text { prospective } \\
\text { cohort }\end{array}$ & $\begin{array}{l}\text { Symptoms } \\
\text { of CMD }\end{array}$ & $\begin{array}{l}\text { Professional rugby } \\
\text { union players; } \\
\text { mean: age 26; career } \\
\text { duration 6; } \\
\text { Australia, England, } \\
\text { France, Ireland, } \\
\text { Italy, New Zealand, } \\
\text { Pacific Islands, } \\
\text { South Africa and } \\
\text { Wales }\end{array}$ & $\begin{array}{l}595 \text { at baseline } \\
\text { and } 333 \text { at follow } \\
\text { up; } 100 / 0\end{array}$ & $\begin{array}{l}\text { GHQ } \\
\text { method }\end{array}$ & 3 & $32 \%$ & $28 \%$ & $\begin{array}{l}\text { Adverse } \\
\text { life events; } \\
\text { rugby } \\
\text { career } \\
\text { dissatisfac } \\
\text { tion }\end{array}$ & $\begin{array}{l}\text { GHQ } \\
\text { score }\end{array}$ & - & $\begin{array}{l}\text { At baseline, there was } 32 \% \\
\text { prevalence of anxiety/depression; } \\
\text { there was a } 28 \% \text { incidence of } \\
\text { anxiety/depression during the } 12- \\
\text { month follow up. Although not } \\
\text { statistically significant, recent } \\
\text { adverse life events or career } \\
\text { dissatisfaction were associated } \\
\text { with the incidence of anxiety/ } \\
\text { depression. }\end{array}$ \\
\hline $\begin{array}{l}\text { Hulley et al., } \\
2007^{[18]}\end{array}$ & $\begin{array}{l}\text { Observation } \\
\text { al, cross- } \\
\text { sectional }\end{array}$ & $\begin{array}{l}\text { Eating } \\
\text { disorders in } \\
\text { elite female } \\
\text { distance } \\
\text { runners }\end{array}$ & $\begin{array}{l}\text { Elite distance } \\
\text { runners from the } \\
\text { UK and Kenya aged } \\
\text { between 15-30 (20.8 } \\
\pm 3.7)\end{array}$ & $\begin{array}{l}\text { Athletes (82 UK } \\
\text { and } 75 \text { Kenyan) } \\
\text { and Non-athlete } \\
\text { controls (97 UK } \\
\text { and } 101 \\
\text { Kenyan); 0/100 }\end{array}$ & $\begin{array}{l}\text { Likert- } \\
\text { scale }\end{array}$ & - & - & - & - & - & $\begin{array}{l}\text { UK athletes } \\
\text { and cont } \\
\text { rols }(20.6 \text {; } \\
21.4) ; \\
\text { Kenyan } \\
\text { athletes and } \\
\text { control } \\
(15.9 ; 17.3)\end{array}$ & $\begin{array}{l}\text { Kenyan athletes scored lower than } \\
\text { UK groups on the GHQ; athletes } \\
\text { scored lower than the controls. } \\
\text { Kenyan runners were less likely to } \\
\text { report symptoms of } \\
\text { anxiety/depression than UK } \\
\text { runners. }\end{array}$ \\
\hline $\begin{array}{l}\text { Ivarsson et } \\
\text { al., 2015 }\end{array}$ & $\begin{array}{l}\text { Observation } \\
\text { al, } \\
\text { prospective } \\
\text { cohort }\end{array}$ & $\begin{array}{l}\text { Predictive } \\
\text { ability of } \\
\text { perceived } \\
\text { talent devel } \\
\text { opment } \\
\text { environmen } \\
\mathrm{t} \text { (TDE) on } \\
\text { well-being } \\
\end{array}$ & $\begin{array}{l}\text { Elite Swedish youth } \\
\text { footballers aged 13- } \\
16 \text { years old (14.16 } \pm \\
1.00)\end{array}$ & $195 ; 100 / 0$ & $\begin{array}{l}\text { Likert- } \\
\text { scale }\end{array}$ & - & - & - & $\begin{array}{l}\text { GHQ } \\
\text { score }\end{array}$ & $\begin{array}{l}\text { TDEQ } \\
\text { (Quality of } \\
\text { talent } \\
\text { developm } \\
\text { ental } \\
\text { environme } \\
\text { nt) }\end{array}$ & - & $\begin{array}{l}\text { A high quality TDE appears to be } \\
\text { associated with higher levels of } \\
\text { self-reported wellbeing among } \\
\text { youth football players; players } \\
\text { experiencing the lowest perceived } \\
\text { TDE quality reported the lowest } \\
\text { level of well-being over time. }\end{array}$ \\
\hline $\begin{array}{l}\text { Kilic et al., } \\
2017^{[19]}\end{array}$ & $\begin{array}{l}\text { Observation } \\
\text { al, cross- } \\
\text { sectional }\end{array}$ & $\begin{array}{l}\text { Symptoms } \\
\text { of common } \\
\text { mental } \\
\text { disorders } \\
\text { and related } \\
\text { stressors }\end{array}$ & $\begin{array}{l}\text { Professional football } \\
\text { and handball } \\
\text { players (current and } \\
\text { retired); Age } \\
\text { current/retired } \\
\text { football players } \\
(25.8 \pm 4.9 / 34.0 \pm \\
4.9) ; \text { Age } \\
\text { current/retired } \\
\text { handball players } \\
(25.3 \pm 4.5 / 35.0 \pm \\
5.6) ; \text { Denmark }\end{array}$ & $\begin{array}{l}348 \text { current \& } \\
345 \text { retired } \\
\text { football players ; } \\
232 \text { current \& } \\
230 \text { retired hand } \\
\text { ball players; } \\
\text { 82/18 (Current } \\
\text { foot ballers); } \\
79 / 21 \text { (Retired } \\
\text { foot ballers); } \\
51 / 49 \text { (Current } \\
\text { hand ball } \\
\text { players); 100/0 } \\
\text { (Retired } \\
\text { handball } \\
\text { players) }\end{array}$ & $\begin{array}{l}\text { GHQ } \\
\text { method }\end{array}$ & 2 & $\begin{array}{l}18 \% \\
\text { (current } \\
\text { footballers); } \\
19 \% \\
\text { (retired } \\
\text { footballers); } \\
26 \% \\
\text { (current } \\
\text { handball } \\
\text { players); } \\
16 \% \\
\text { (retired } \\
\text { handball } \\
\text { players) }\end{array}$ & - & $\begin{array}{l}\text { Severe } \\
\text { injuries; } \\
\text { surgeries*; } \\
\text { recent } \\
\text { adverse } \\
\text { life } \\
\text { events* }\end{array}$ & $\begin{array}{l}\text { GHQ } \\
\text { score }\end{array}$ & - & $\begin{array}{l}\text { A prevalence of } 18 \% \text { and } 19 \% \\
\text { anxiety/depression was observed } \\
\text { for current and retired football } \\
\text { players respectively; a prevalence } \\
\text { of } 26 \% \text { and } 16 \% \text { anxiety/ } \\
\text { depression was observed for } \\
\text { current and retired handball } \\
\text { players respectively; significant } \\
\text { associations between a higher } \\
\text { number of recent adverse life } \\
\text { events and risk of anxiety/ } \\
\text { depression in all athletes; in } \\
\text { retired football players there were } \\
\text { significant associations with a } \\
\text { higher number of severe injuries } \\
\text { and surgeries with risk of } \\
\text { anxiety/depression. }\end{array}$ \\
\hline
\end{tabular}


Table 1 continued. Summary of General Health Questionnaire (GHQ) studies on athletes

\begin{tabular}{|c|c|c|c|c|c|c|c|c|c|c|c|c|}
\hline Author & Study design & Purpose & $\begin{array}{l}\text { Population; } \\
\text { Country }\end{array}$ & $\begin{array}{l}\text { N; Men/ } \\
\text { Women \% }\end{array}$ & $\begin{array}{l}\text { GHQ } \\
\text { scoring } \\
\text { method }\end{array}$ & $\begin{array}{l}\text { GHQ } \\
\text { cut off } \\
\text { point } \\
\end{array}$ & Prevalence & Incidence & Predictor & Outcome & $\begin{array}{l}\text { GHQ } \\
\text { mean } \\
\text { score }\end{array}$ & Findings \\
\hline $\begin{array}{l}\text { Kilic et al., } \\
2018^{[22]}\end{array}$ & $\begin{array}{l}\text { Observational, } \\
\text { prospective } \\
\text { cohort }\end{array}$ & $\begin{array}{l}\text { Severe } \\
\text { musculoskel } \\
\text { etal } \\
\text { time-loss } \\
\text { injuries and } \\
\text { symptoms } \\
\text { of common } \\
\text { mental } \\
\text { disorders }\end{array}$ & $\begin{array}{l}\text { Professional } \\
\text { footballers from } \\
\text { national player } \\
\text { unions; Age ( } 27 \pm \\
\text { 5); duration of } \\
\text { career (8 } \pm 5) \text {; } \\
\text { Finland, France, } \\
\text { Norway, Spain, } \\
\text { Sweden }\end{array}$ & $\begin{array}{l}384 \text { at } \\
\text { baseline and } \\
262 \text { at follow } \\
\text { up; } 100 / 0\end{array}$ & $\begin{array}{l}\text { GHQ } \\
\text { method }\end{array}$ & 3 & $32 \%$ & - & $\begin{array}{l}\text { GHQ score; } \\
\text { Musculoske } \\
\text { letal time- } \\
\text { loss injuries }\end{array}$ & $\begin{array}{l}\text { Musculos } \\
\text { keletal } \\
\text { time-loss } \\
\text { injuries; } \\
\text { GHQ } \\
\text { score }\end{array}$ & - & $\begin{array}{l}32 \% \text { prevalence of } \\
\text { anxiety/depression at baseline; } \\
\text { not associated with the incidence } \\
\text { of severe musculoskeletal time- } \\
\text { loss injury. However, } \\
\text { musculoskeletal injuries at } \\
\text { baseline were associated with the } \\
\text { incidence of anxiety/depression } \\
\text { in the follow up period even } \\
\text { when adjusted for age and } \\
\text { adverse life events. }\end{array}$ \\
\hline $\begin{array}{l}\text { Niazi et al., } \\
2014[20]\end{array}$ & $\begin{array}{l}\text { Observational, } \\
\text { cross-sectional }\end{array}$ & $\begin{array}{l}\text { Relationship } \\
\text { between } \\
\text { emotional } \\
\text { intelligence } \\
\text { and mental } \\
\text { health in } \\
\text { collegiate } \\
\text { champions }\end{array}$ & $\begin{array}{l}\text { Collegiate athletes } \\
\text { of Islamic Azad } \\
\text { University; Age } \\
(22.35 \pm 2.49) \text {; } \\
\text { history of sports in } \\
\text { years }(9 \pm 2) \text {; } \\
\text { various sports; } \\
\text { Iran }\end{array}$ & $192 ; \mathrm{NS}$ & NS & NS & - & - & Self-control ${ }^{*}$ & $\begin{array}{l}\text { GHQ } \\
\text { score }\end{array}$ & - & $\begin{array}{l}\text { Significant positive correlation } \\
\text { between emotional intelligence } \\
\text { and mental health; self-control } \\
\text { explains } 76 \% \text { of the variations in } \\
\text { mental health. }\end{array}$ \\
\hline $\begin{array}{l}\text { Noblet et al., } \\
2003^{[21]}\end{array}$ & $\begin{array}{l}\text { Observational, } \\
\text { cross-sectional }\end{array}$ & $\begin{array}{l}\text { Predictors of } \\
\text { the strain } \\
\text { experienced } \\
\text { by } \\
\text { professional } \\
\text { Australian } \\
\text { Footballers } \\
\text { (Aussie } \\
\text { rules) }\end{array}$ & $\begin{array}{l}\text { Professional } \\
\text { Australian football } \\
\text { players }\end{array}$ & $255 ; 100 / 0$ & $\begin{array}{l}\text { Likert- } \\
\text { scale }\end{array}$ & - & - & - & $\begin{array}{l}\text { Post- } \\
\text { football } \\
\text { uncertainty* } \\
\text {; social } \\
\text { support } \\
\text { from work*; } \\
\text { pressure to } \\
\text { perform* }\end{array}$ & $\begin{array}{l}\text { GHQ } \\
\text { score }\end{array}$ & - & $\begin{array}{l}\text { Post-football uncertainty, social } \\
\text { support from work and the } \\
\text { constant pressure to perform } \\
\text { were significant predictors of } \\
\text { psychological health. }\end{array}$ \\
\hline $\begin{array}{l}\text { Peretti-Watel } \\
\text { et al., 2004 }\end{array}$ & $\begin{array}{l}\text { Observational, } \\
\text { cross-sectional }\end{array}$ & $\begin{array}{l}\text { Risky } \\
\text { behaviour } \\
\text { among elite } \\
\text { student } \\
\text { athletes }\end{array}$ & $\begin{array}{l}\text { Elite student } \\
\text { athletes competing } \\
\text { at regional, } \\
\text { national or } \\
\text { international/Oly } \\
\text { mpic level; age } \\
\text { (18.3); France }\end{array}$ & $458 ; 65 / 35$ & $\begin{array}{l}\text { Likert- } \\
\text { scale }\end{array}$ & - & - & - & $\begin{array}{l}\text { Smoking; } \\
\text { Cannabis } \\
\text { use }\end{array}$ & $\begin{array}{l}\text { GHQ } \\
\text { score }\end{array}$ & $\begin{array}{l}\text { Girls } \\
(15.2 \pm \\
6.9) ; \text { Boys } \\
(11.3 \pm \\
5.3)\end{array}$ & $\begin{array}{l}\text { Girls had a significantly higher } \\
\text { GHQ score than boys. GHQ } \\
\text { scores were significantly } \\
\text { correlated with smoking and } \\
\text { cannabis use among elite student } \\
\text { athletes. }\end{array}$ \\
\hline $\begin{array}{l}\text { Quarrie et } \\
\text { al., 2001 }\end{array}$ & $\begin{array}{l}\text { Observational, } \\
\text { prospective } \\
\text { cohort }\end{array}$ & $\begin{array}{l}\text { Association } \\
\text { between } \\
\text { potential } \\
\text { risk factors } \\
\text { and injury } \\
\text { risk }\end{array}$ & $\begin{array}{l}\text { Professional rugby } \\
\text { union players; age } \\
(20.6 \pm 3.7) ; \mathrm{New} \\
\text { Zealand }\end{array}$ & $250 ; 100 / 0$ & NS & NS & - & - & GHQ score & Injury & - & $\begin{array}{l}\text { No significant association of } \\
\text { GHQ score with injury risk. }\end{array}$ \\
\hline
\end{tabular}


Table 1 continued. Summary of General Health Questionnaire (GHQ) studies on athletes

\begin{tabular}{|c|c|c|c|c|c|c|c|c|c|c|c|c|}
\hline Author & Study design & Purpose & $\begin{array}{l}\text { Population; } \\
\text { Country }\end{array}$ & $\begin{array}{l}\text { N; Men/ } \\
\text { Women \% }\end{array}$ & $\begin{array}{l}\text { GHQ } \\
\text { scoring } \\
\text { method }\end{array}$ & $\begin{array}{l}\text { GHQ } \\
\text { cut off } \\
\text { point }\end{array}$ & Prevalence & Incidence & Predictor & Outcome & $\begin{array}{l}\text { GHQ mean } \\
\text { score }\end{array}$ & Findings \\
\hline $\begin{array}{l}\text { Schuring } \\
\text { et al., } \\
2017 a^{[23]}\end{array}$ & $\begin{array}{l}\text { Observational } \\
\text {, cross- } \\
\text { sectional }\end{array}$ & $\begin{array}{l}\text { Association } \\
\text { between } \\
\text { osteoarthritis } \\
(\mathrm{OA}) \text { and } \\
\text { symptoms of } \\
\text { common } \\
\text { mental } \\
\text { disorders }\end{array}$ & $\begin{array}{l}\text { Former elite } \\
\text { athletes (rugby, } \\
\text { football, ice } \\
\text { hockey, Gaelic } \\
\text { sports and cricket); } \\
\text { age ( } 37 \pm 6) \text {; } \\
\text { duration of career } \\
(10 \pm 5) \text {; duration of } \\
\text { retirement }(6 \pm 5) ; \\
\text { Finland, France, } \\
\text { Ireland, Norway, } \\
\text { South Africa, } \\
\text { Spain, Sweden and } \\
\text { Switzerland }\end{array}$ & $\begin{array}{l}\text { With OA } \\
(200) \text { and } \\
\text { Without OA } \\
(402) ; 100 / 0\end{array}$ & $\begin{array}{l}\text { GHQ } \\
\text { method }\end{array}$ & 4 & $\begin{array}{l}31 \% \text { (with } \\
\text { OA); } 25 \% \\
\text { (without } \\
\text { OA) }\end{array}$ & - & $\mathrm{OA}^{*}$ & $\begin{array}{l}\text { GHQ } \\
\text { score }\end{array}$ & - & $\begin{array}{l}\text { OA might be a risk factor for } \\
\text { developing symptoms of } \\
\text { CMD in former elite athletes. } \\
\text { Prevalence of } 31 \% \text { and } 25 \% \\
\text { for retired athletes with OA } \\
\text { and those without OA } \\
\text { respectively. OA was } \\
\text { significantly associated with } \\
\text { having more than } 2 \text { CMDs. } \\
\text { Further, there was a } \\
\text { significant association of OA } \\
\text { with anxiety/depression in } \\
\text { former ice hockey players. }\end{array}$ \\
\hline $\begin{array}{l}\text { Schuring } \\
\text { et al., } \\
2017 b^{[44]}\end{array}$ & $\begin{array}{l}\text { Observational } \\
\text {, prospective } \\
\text { cohort }\end{array}$ & $\begin{array}{l}\text { Mental well- } \\
\text { being of } \\
\text { current and } \\
\text { retired } \\
\text { professional } \\
\text { cricketers }\end{array}$ & $\begin{array}{l}\text { Current (age: } 27 \pm \\
\text { 5) and former } \\
\text { professional } \\
\text { cricketers (age: } 36 \pm \\
\text { 6); duration of } \\
\text { career (current: } 6 \pm \\
\text { 5), (former: } 12 \pm 6 \text { ); } \\
\text { South Africa }\end{array}$ & $\begin{array}{l}116: 78 \\
\text { (current) } \\
\text { and 38 } \\
\text { (former) at } \\
\text { baseline; } \\
91 / 9\end{array}$ & $\begin{array}{l}\text { GHQ } \\
\text { method }\end{array}$ & 2 & $\begin{array}{l}37 \% \\
\text { (current); } \\
24 \% \\
\text { (retired) }\end{array}$ & $\begin{array}{l}15 \% \\
\text { (current) }\end{array}$ & $\begin{array}{l}\text { Significant } \\
\text { injuries; } \\
\text { surgeries; } \\
\text { adverse life } \\
\text { events*; career } \\
\text { dissatisfaction } \\
\text { * }\end{array}$ & $\begin{array}{l}\text { GHQ } \\
\text { score }\end{array}$ & - & $\begin{array}{l}\text { Prevalence of } 37 \% \text { and } 15 \% \\
\text { incidence over } 6 \text { months for } \\
\text { anxiety/ depression in } \\
\text { current cricketers; } \\
\text { prevalence of } 24 \% \text { for } \\
\text { anxiety/ depression in } \\
\text { former cricketers. Career } \\
\text { dissatisfaction and adverse } \\
\text { life events had positive } \\
\text { associations with anxiety/ } \\
\text { depression in current } \\
\text { cricketers. }\end{array}$ \\
\hline $\begin{array}{l}\text { Sekizaki } \\
\text { et al., } \\
2017^{[45]}\end{array}$ & $\begin{array}{l}\text { Randomised } \\
\text { controlled } \\
\text { trial }\end{array}$ & $\begin{array}{l}\text { Effectiveness } \\
\text { and } \\
\text { contribution } \\
\text { of internet- } \\
\text { based } \\
\text { Cognitive } \\
\text { Behavioural } \\
\text { Therapy } \\
\text { (iCBT) to } \\
\text { mental } \\
\text { healthcare in a } \\
\text { school setting }\end{array}$ & $\begin{array}{l}\text { High school } \\
\text { athletes competing } \\
\text { at a national level } \\
\text { in various sports; } \\
\text { Japan }\end{array}$ & $\begin{array}{l}80: 40 \\
\text { intervention } \\
\text { group and } \\
40 \text { controls; } \\
100 / 0\end{array}$ & $\begin{array}{l}\text { GHQ } \\
\text { method }\end{array}$ & NS & - & - & - & - & $\begin{array}{l}\text { Pre: } \\
\text { Intervention } \\
\text { and control } \\
(3 ; 3.3) ; \text { Post: } \\
\text { Intervention } \\
\text { and control } \\
(1.6 ; 2.2)\end{array}$ & $\begin{array}{l}\text { School mental healthcare } \\
\text { programme using iCBT is } \\
\text { suitable for students and } \\
\text { useful for coping with stress } \\
\text { and reducing depressed } \\
\text { mood and anxiety (lower } \\
\text { GHQ scores) in young } \\
\text { people, especially athletes. }\end{array}$ \\
\hline
\end{tabular}


REVIEW

Table 1 continued. Summary of General Health Questionnaire (GHQ) studies on athletes

\begin{tabular}{|c|c|c|c|c|c|c|c|c|c|c|c|c|}
\hline Author & Study design & Purpose & $\begin{array}{l}\text { Population; } \\
\text { Country }\end{array}$ & $\begin{array}{l}\text { N; Men/ } \\
\text { Women \% }\end{array}$ & $\begin{array}{l}\text { GHQ } \\
\text { scoring } \\
\text { method }\end{array}$ & $\begin{array}{l}\text { GHQ } \\
\text { cut off } \\
\text { point }\end{array}$ & Prevalence & Incidence & Predictor & Outcome & $\begin{array}{l}\text { GHQ } \\
\text { mean } \\
\text { score }\end{array}$ & Findings \\
\hline $\begin{array}{l}\text { Stephan, } \\
2003^{[33]}\end{array}$ & $\begin{array}{l}\text { Observational, } \\
\text { prospective } \\
\text { cohort }\end{array}$ & $\begin{array}{l}\text { Repercussions } \\
\text { of transition } \\
\text { out of elite } \\
\text { sport on } \\
\text { subjective } \\
\text { well-being }\end{array}$ & $\begin{array}{l}\text { Olympic } \\
\text { athletes (active } \\
\text { and retired); } \\
\text { athletes ages } \\
\text { ranged from } 27 \\
\text { to } 35 \text { years } \\
\text { (retired: } 31 \pm 4 \text {; } \\
\text { active: } 29 \pm 2 \text { ); } \\
\text { France }\end{array}$ & $\begin{array}{l}32: 16 \text { retired } \\
\text { and } 16 \text { active; } \\
50 / 50\end{array}$ & $\begin{array}{l}\text { Likert- } \\
\text { scale }\end{array}$ & - & - & - & - & - & $\begin{array}{l}\text { Time 1, } \\
2,3: \\
25.06 ; \\
21.18 \\
19.56\end{array}$ & $\begin{array}{l}\text { At the time of retirement, retired } \\
\text { athletes exhibited significantly } \\
\text { decreased subjective well-being (mean } \\
\text { GHQ score = 25.06) compared to } \\
\text { active athletes (mean GHQ score = } \\
\text { 21.18). However, as transitional } \\
\text { athletes became used to retirement, } \\
\text { their well-being increased over time } \\
\text { (mean GHQ score = 19.56). }\end{array}$ \\
\hline $\begin{array}{l}\text { Totterdell et } \\
\text { al., 2001 }\end{array}$ & $\begin{array}{l}\text { Observational, } \\
\text { cross-sectional }\end{array}$ & $\begin{array}{l}\text { Negative } \\
\text { mood } \\
\text { regulation } \\
\text { (NMR) } \\
\text { expectancies } \\
\text { and sports } \\
\text { performance }\end{array}$ & $\begin{array}{l}\text { Professional } \\
\text { cricketers from } \\
\text { county cricket } \\
\text { clubs; ages } \\
\text { ranged from } 17 \\
\text { to } 38 \text { years ( } 25 \\
\pm 5 \text {; England }\end{array}$ & $46 ; 100 / 0$ & $\begin{array}{l}\text { Likert- } \\
\text { scale }\end{array}$ & - & - & - & - & - & $\begin{array}{l}10.04 \pm \\
5.21\end{array}$ & $\begin{array}{l}\text { Mean GHQ score (10.04) was not } \\
\text { significantly correlated with } \\
\text { performance over a season; it was } \\
\text { significantly correlated with NMR, } \\
\text { which itself is significantly correlated } \\
\text { with performance. Furthermore, NMR } \\
\text { did not correlate with performance } \\
\text { when well-being was covaried for. }\end{array}$ \\
\hline $\begin{array}{l}\text { van Ramele } \\
\text { et al., 2017 }\end{array}$ & $\begin{array}{l}\text { Observational, } \\
\text { prospective } \\
\text { cohort }\end{array}$ & $\begin{array}{l}\text { Incidence of } \\
\text { symptoms of } \\
\text { common } \\
\text { mental } \\
\text { disorders }\end{array}$ & $\begin{array}{l}\text { Retired } \\
\text { professional } \\
\text { footballers; age } \\
(35 \pm 6) ; \\
\text { duration of } \\
\text { career }(12 \pm 5) \text {; } \\
\text { duration of } \\
\text { retirement }(4 \pm \\
\text { 3); Various }\end{array}$ & $\begin{array}{l}212 \text { (baseline) } \\
\text { and } 194 \\
\text { (follow up); } \\
100 / 0\end{array}$ & $\begin{array}{l}\text { GHQ } \\
\text { method }\end{array}$ & 3 & - & $29 \%$ & $\begin{array}{l}\text { Adverse } \\
\text { life events }\end{array}$ & $\begin{array}{l}\text { GHQ } \\
\text { score }\end{array}$ & - & $\begin{array}{l}\text { Highest incidence over } 12 \text { months was } \\
\text { anxiety/depression }(29 \%) \text {. However, } \\
\text { there was no association between } \\
\text { adverse life events and } \\
\text { anxiety/depression. } 96 \% \text { of retired } \\
\text { football players agreed that mental } \\
\text { health can influence players during } \\
\text { their career while more than half } \\
\text { agreed that mental health affected } \\
\text { their performance. More than } 80 \% \\
\text { agreed that there is insufficient } \\
\text { medical support for mental health for } \\
\text { both current and retired footballers }\end{array}$ \\
\hline $\begin{array}{l}\text { Wilson et al., } \\
2015^{[46]}\end{array}$ & $\begin{array}{l}\text { Quasi- } \\
\text { experimental }\end{array}$ & $\begin{array}{l}\text { Effects of a } \\
\text { diet and } \\
\text { exercise } \\
\text { intervention } \\
\text { on body } \\
\text { composition, } \\
\text { metabolism, } \\
\text { bone and } \\
\text { mental health }\end{array}$ & $\begin{array}{l}\text { Highly } \\
\text { experienced } \\
\text { jockeys; UK }\end{array}$ & 10; NS & $\begin{array}{l}\text { GHQ } \\
\text { method }\end{array}$ & 4 & $21 \%$ & - & - & - & $\begin{array}{l}\text { Pre: } 10.3 \\
\pm 4.3 ; \\
\text { Post: } 8.9 \\
\pm 3.8\end{array}$ & $\begin{array}{l}\text { There was a } 21 \% \text { prevalence of jockeys } \\
\text { suffering from symptoms of } \\
\text { anxiety/depression. Mean GHQ-12 } \\
\text { score was } 10.3 \text { prior to the dietary } \\
\text { intervention reducing to } 8.9 \text { post- } \\
\text { intervention. However, this was not } \\
\text { statistically significant. }\end{array}$ \\
\hline
\end{tabular}


Conflict of interest and source of funding: The authors declare no conflict of interest and no source of funding.

\section{Author contributions:}

All authors conceptualised the need for a review. NA performed the literature search and synthesis. NA drafted the manuscript. All authors provided critical input and approved the final version.

\section{References}

1. Gulliver A, Griffiths KM, Mackinnon A, et al. The mental health of Australian elite athletes. J Sci Med Sport 2015;18(3):255-261. [doi: 10.1016/j.jsams.2014.04.006][PMID: 24882147]

2. Heun R, Pringle A. Football does not improve mental health : a systematic review on football and mental health disorders. Global Psychiatry 2018;1(1):25-38. [doi:10.2478/gp-2018-0001]

3. Reardon CL, Factor RM. Sport psychiatry:A systematic review of diagnosis and medical treatment of mental illness in athletes. Sport Med 2010;40(11):961-980. [doi:10.2165/11536580000000000-00000][PMID: 20942511]

4. Rice SM, Purcell R, De Silva S, et al. The mental health of elite athletes: A narrative systematic review. Sports Med 2016;46(9):1333-1353. [doi:10.1007/s40279-016-0492-2][PMID: 26896951]

5. Wolanin A, Gross M, Hong E. Depression in athletes: prevalence and risk factors. Curr Sports Med Rep 2015;14(1):5660. [doi:10.1249/JSR.0000000000000123][PMID: 25574886]

6. Reardon CL, Hainline B, Aron CM, et al. Mental health in elite athletes: International Olympic Committee consensus statement (2019). Br J Sports Med 2019;53(11):667-699. [doi:10.1136/bjsports-2019-100715][PMID: 31097450]

7. Han DH, McDuff D, Thompson D, et al. Attentiondeficit/hyperactivity disorder in elite athletes: a narrative review. Br J Sports Med 2019;53(12):741-745. [doi:10.1136/bjsports-2019-100713]

8. Goldberg DP. The detection of psychiatric illness by questionnaire: A technique for the identification and assessment of non-psychotic psychiatric illness. Psychol Med 1973;3(2):257. [doi:10.1017/S0033291700048613]

9. Foskett RL, Longstaff F. The mental health of elite athletes in the United Kingdom. J Sci Med Sport 2018;21(8):765-770. [doi:10.1016/j.jsams.2017.11.016][PMID:29289498]

10. Gnambs T, Staufenbiel T. The structure of the General Health Questionnaire (GHQ-12): two meta-analytic factor analyses. Health Psychol Rev 2018; 12(2):179-194.[doi: 10.1080/17437199.2018.1426484] [PMID: 29325498]

11. Goldberg DP, Gater R, Sartorius N, et al. The validity of two versions of the GHQ in the WHO study of mental illness in general health care. Psychol Med 1997;27(1):191-197. [doi: 10.1017/s0033291796004242]PMID: 9122299]

12. Goldberg DP, Hillier VF. A scaled version of the Genral Health Questionnaire. Psychol Med 1979;9(1):139-145. [doi: 10.1017/S0033291700021644] [PMID: 424481]

13. Goldberg DP, Williams P. A user's guide to the General Health Questionnaire. 1988. NFER-NELSON, London.

14. Rey JJ, Abad FJ, Barrada JR, et al. The impact of ambiguous response categories on the factor structure of the GHQ-12. Psychol Assess 2014;26(3):1021-1030. [doi:10.1037/a0036468] [PMID: 24708083]

15. Goldberg DP, Oldehinkel T, Ormel J. Why GHQ threshold varies from one place to another. Psychol Med 1998;28(4):915921. [doi:10.1017/S0033291798006874][PMID: 9723146]

16. Gouttebarge V, Aoki H, Ekstrand J, et al. Are severe musculoskeletal injuries associated with symptoms of common mental disorders among male European professional footballers? Knee Surg Sport Traumatol Arthrosc 2015;24(12):3934-3942. [doi: 10.1007/s00167-015-3729-y][PMID: 26233596]

17. Gouttebarge V, Kerkhoffs G, Lambert M. Prevalence and determinants of symptoms of common mental disorders in retired professional Rugby Union players. Eur J Sport Sci 2016;16(5):595-602.

[doi:10.1080/17461391.2015.1086819][PMID: 26419657]

18. Hulley A, Currie A, Njenga F, et al. Eating disorders in elite female distance runners: Effects of nationality and running environment. Psychol Sport Exerc 2007;8(4):521-533. [doi.org/10.1016/j.psychsport.2006.07.001]

19. Kilic Ö, Aoki H, Haagensen R, et al. Symptoms of common mental disorders and related stressors in Danish professional football and handball. Eur J Sport Sci 2017;17(10):13281334.[doi:10.1080/17461391.2017.1381768][PMID: 28961069]

20. Niazi S, Bai N, Gharaei S. The relationship between emotional intelligence and mental health in collegiate champions. Int Res J Manag Sci 2014;2(1):12-15. [doi:10.1080/10413200305394]

21. Noblet A, Rodwell J, McWilliams J. Predictors of the strain experienced by professional Australian footballers. J Appl Sport Psychol 2003;15(2):184-193. [doi:10.1080/10413200305394]

22. Peretti-Watel P, Guagliardo V, Verger P, et al. Risky behaviours among young elite-student-athletes: results from a pilot survey in south-eastern France. Int Rev Sociol Sport 2004;39(2):233-244. [doi:10.1177/1012690204043467]

23. Schuring N, Aoki H, Gray J, et al. Osteoarthritis is associated with symptoms of common mental disorders among former elite athletes. Knee Surg Sport Traumatol Arthrosc 2017;25(10):31793185.[ doi:10.1007/s00167-016-4255-2][PMID: 27488101]

24. Totterdell P, Leach D. Negative mood regulation expectancies and sports performance: An investigation involving professional cricketers. Psychol Sport Exerc 2001;2(4):249-265. [doi:10.1016/S1469-0292(01)00016-4]

25. Brown JC, Kerkhoffs G, Lambert MI, Gouttebarge V. Forced retirement from professional rugby union is associated with symptoms of distress. Int J Sports Med 2017;38(8):582-587. [doi:10.1055/s-0043-103959][PMID: 28564743]

26. Gouttebarge V, Aoki H, Kerkhoffs G. Symptoms of common mental disorders and adverse health behaviours in male professional soccer players. J Hum Kinet 2015;49(1):277-286. [doi:10.1515/hukin-2015-0130][PMID: 26925182]

27. Gouttebarge V, Aoki H, Kerkhoffs G. Prevalence and determinants of symptoms related to mental disorders in retired male professional footballers. J Sport Med Phys Fitness 2016;56(5), 648-654. [PMID: 27285354]

28. Gouttebarge V, Aoki H, Lambert $M$, et al. A history of concussions is associated with symptoms of common mental disorders in former male professional athletes across a range of sports. Phys Sportsmed 2017;45(4):443-449. [doi: 10.1080/00913847.2017.1376572] [PMID: 28870119]

29. Gouttebarge V, Aoki H, Verhagen E, et al. Are level of education and employment related to symptoms of common mental disorders in current and retired professional footballers? Asian J Sports Med 2016;7(2):e28447. [doi:10.5812/asjsm.28447] [PMID: 27625749]

30. Gouttebarge V, Backx FJ, Aoki H, Kerkhoffs G. Symptoms of common mental disorders in professional football (soccer) across five European countries. J Sports Sci Med 2015;14(4):811-818. [PMID: 26664278]

31. Gouttebarge V, Frings-Dresen MH, Sluiter JK. Mental and psychosocial health among current and former professional footballers. Occup Med (London) 2015;65(3):190-196. [doi:10.1093/occmed/kqu202][PMID: 25638208]

32. Gouttebarge V, Jonkers $\mathrm{R}$, Moen $\mathrm{M}$, et al. The prevalence and risk 
indicators of symptoms of common mental disorders among current and former Dutch elite athletes. J Sports Sci 2017;35(21):2148-2156.

[doi:10.1080/02640414.2016.1258485][PMID: 27894209]

33. Blakelock DJ, Chen MA, Prescott T. Psychological distress in elite adolescent soccer players following deselection. J Clin Sport Psychol 2016;10(1):59-77. [doi:10.1123/jcsp.2015-0010]

34. Gouttebarge V, Aoki H, Verhagen E, et al. A 12-month prospective cohort study of symptoms of common mental disorders among European professional footballers. Clin J Sport Med 2017;27(5):487-492. [doi:10.1097/JSM.0000000000000388] [PMID: 27623187]

35. Stephan Y, Bilard J, Ninot G, et al. Repercussions of transition out of elite sport on subjective well-being: A one-year study. J Appl Sport Psychol 2003; 4 4(4):354-371. [doi:10.1080/10413200390238022]

36. van Ramele S, Aoki H, Kerkhoffs GM, et al. Mental health in retired professional football players: 12-month incidence, adverse life events and support. Psychol Sport Exerc 2017;28:8590. [doi:10.1016/j.psychsport.2016.10.009]

37. Gouttebarge V, Hopley P, Kerkhoffs G, et al. A 12-month prospective cohort study of symptoms of common mental disorders among professional rugby players. Eur J Sport Sci 2018:; 18(7): 1004-1012.[ doi:10.1080/17461391.2018.1466914] [PMID: 29698129]

38. Gouttebarge V, Jonkers R, Moen M, et al. A prospective cohort study on symptoms of common mental disorders among Dutch elite athletes. Phys Sportsmed 2017;45(4):426-432. [doi:10.1080/00913847.2017.1370987][PMID: 28826314]

39. Gouttebarge V, Kerkhoffs G. A prospective cohort study on symptoms of common mental disorders among current and retired professional ice hockey players. Phys Sportsmed 2017;45(3):252-258. [doi:10.1080/00913847.2017.1338497][PMID: 28576114]

40. Gouttebarge V, Tol JL, Kerkhoffs G. Epidemiology of symptoms of common mental disorders among elite Gaelic athletes: a prospective cohort study. Phys Sportsmed 2016;44(3):283-289. [doi: 10.1080/00913847.2016.1185385][PMID: 27149248]

41. Ivarsson A, Stenling A, Fallby J, et al. The predictive ability of the talent development environment on youth elite football players' well-being: A person-centered approach. Psychol Sport Exerc 2015;16(1):15-23. [doi:10.1016/j.psychsport.2014.09.006]

42. Kiliç Ö, Aoki H, Goedhart E, et al. Severe musculoskeletal timeloss injuries and symptoms of common mental disorders in professional soccer: a longitudinal analysis of 12-month followup data. Knee Surg Sport Traumatol Arthrosc 2018;26(3):946-954. [doi:10.1007/s00167-017-4644-1][PMID: 28698928]

43. Quarrie KL, Alsop JC, Waller AE, et al. The New Zealand rugby injury and performance project. VI. A prospective cohort study of risk factors for injury in rugby union football. Br J Sports Med 2001;35(3):157-166. [doi:10.1136/bjsm.35.3.157] [PMID: 11375873]

44. Schuring N, Kerkhoffs G, Gray J, et al. The mental wellbeing of current and retired professional cricketers: an observational prospective cohort study. Phys Sportsmed 2017;45(4):463-469. [doi:10.1080/00913847.2017.1386069][PMID: 28952405]

45. Sekizaki R, Nemoto T, Tsujino N, et al. School mental healthcare services using internet-based cognitive behaviour therapy for young male athletes in Japan. Early Interv Psychiatry 2019;13(1),79-85. [doi: 10.1111/eip.12454][PMID: 28613407]

46. Wilson G, Pritchard PP, Papageorgiou C, et al. Fasted exercise and increased dietary protein reduces body fat and improves strength in jockeys. Int J Sports Med 2015;36(12):1008-1014. [doi:10.1055/s-0035-1549920][PMID: 26212241]

47. American Psychiatric Association: Diagnostic and statistical manual of mental disorders, 5th ed.:DSM-5 Arlington, VA: American Psychiatric Association; 2013.

48. World Health Organization. Depression and other common mental disorders: global health estimates. Geneva: World Heal Organization 2017. Licence: CC BY-NC-SA 3.0 IGO 